\title{
Current treatment of low grade gliomas
}

\author{
Martin J. van den Bent, Tom J. Snijders, Jacoline E. C. Bromberg \\ Received: 25 March 2012 / Accepted: 27 April 2012 / Published online: 5 June 2012 \\ (C) The Author(s) 2012. This article is published with open access at Springerlink.com
}

\begin{abstract}
Low grade gliomas affect predominantly young adults, and have a relatively favorable prognosis compared to grade III and grade IV gliomas. The challenge for an optimal management of these patients is to find the balance between an optimal survival and the preservation of neurological function including cognition. Because all medical treatments may induce side effects, in young and nearly asymptomatic patients the choices can be difficult. This review summarizes the current strategies: a watch-and-wait policy, surgery, chemotherapy, and radiotherapy.
\end{abstract}

Keywords: Low grade glioma, Astrocytoma, Oligodendroglioma, Temozolomide, Chemotherapy, Surgery, Radiotherapy

\section{Introduction}

The diffuse low grade (WHO grade II) gliomas (LGG) are histologically subdivided in three categories: astrocytoma, oligodendroglioma, and mixed oligoastrocytoma. Because as a rule mixed oligoastrocytoma are characterized by either the presence of TP53 mutations (typical for astrocytoma) or by the presence of a $1 \mathrm{p} / 19 \mathrm{q}$ co-deletion (typical for oligodendroglioma), on the biological level mixed oligoastrocytoma do not appear to reflect a true entity and its name is more indicative for the difficulties of the histological diagnosis of glioma [1]. The optimal treatment of low-grade glioma remains controversial. Guidelines on the early management of young patients presenting with seizures only and a lesion compatible

M. J. van den Bent, MD ( $\bowtie)$

Daniel den Hoed Oncology Center, Neuro-Oncology Unit, PO Box 5201, 3008 AE Rotterdam, The Netherlands e-mail: m.vandenbent@erasmusmc.nl

M. J. van den Bent, MD · J. E. C. Bromberg, MD Erasmus MC-Daniel den Hoed Cancer Center, Rotterdam, The Netherlands

T. J. Snijders, MD

Rudolf Magnus Institute of Neuroscience, Department of Neurology, Utrecht University Medical Center

Utrecht, Utrecht, The Netherlands with an LGG is not based on solid clinical evidence. As such, the patients may do well for a prolonged period of time without any treatment; many physicians defer diagnostic procedures and treatment as long as possible, whereas others advocate early treatment consisting of an extensive resection with or without adjuvant therapy. Arguments against early treatment are derived from the observation that many patients remain asymptomatic (apart from the seizures) for a prolonged period of time, and may deteriorate following treatment [2-5]. Arguments for early treatment are uncertainty about the diagnosis and potentially better survival after early extensive resections [6, 7]. Plus, even so-called stable untreated low-grade glioma show a constant tendency to grow over time (on average $4.1 \mathrm{~mm}$ per year) [8]. This implies that patients followed initially with a watch-and-wait policy will require treatment for a larger lesion once treatment is initiated.

The reliability of a 'low grade glioma' MR diagnosis

Although a typical MRI scan with a nonenhancing T2hyperintense mass will usually harbor an LGG, many exceptions exist: reports indicate that up to $30-45 \%$ of nonenhancing lesions suggestive of LGG turn out to be high-grade glioma [7]. Despite this fact, a biopsy is not necessarily required in all cases of suspected LGGs: in case of a 'watch-and-wait' policy, adequate neuroradiological follow-up will identify patients with progressive lesions requiring histological diagnosis and treatment. Radioactively labeled amino acid PET scanning may help to distinguish between true LGG and histologically high grade but nonenhancing tumors on MR imaging [9]. Growth rate on MRI imaging within the first 6 months of follow-up has also shown to be of prognostic value [10]. Although enhancement is usually indicative of a high grade lesion, LGG (especially oligodendroglioma) can show some minor non-nodular enhancement without an adverse prognostic significance [11]. 
What evidence is available to decide at what moment histological diagnosis should be obtained and treatment should be initiated?

The only prospective clinical trial into early versus delayed treatment is the randomized EORTC trial that showed early radiotherapy improves progression free survival, without affecting overall survival [12]. This study suggested that with respect to survival, the delay in radiotherapy does not adversely affect outcome; however, the quality of life and cognition were not investigated. Regardless of the type of treatment, treatment may induce acute (surgery) and delayed (radiotherapy) neurological toxicities in LGG patients. This may lead to decreased quality of life and cognitive dysfunction $[2,4$, $13,14]$. A recent large but retrospective study on cognitive deficits in low-grade glioma patients observed after many years of follow-up an association between prior radiotherapy and cognitive deficits [2]. In an earlier report on that cohort, the investigators had shown that having a tumor, use of anticonvulsants, and radiotherapy with fraction size exceeding 2 Gy were also associated with cognitive deficits [15].

Can we select patients in which early diagnosis and treatment is indicated?

In patients with focal deficits, raised intracranial pressure or tumors showing rapid radiological progression, the need for immediate treatment is undisputed [16]. Intractable seizures may also constitute an indication for treatment, as treatment may improve seizure control $[12,17,18]$. In general, the proponents for a watch-andwait policy assume that the presence of poor prognostic factors can be used to identify patients that require treatment. Several clinical prognostic factors have been identified, in particular age, size of the lesion, tumor crossing the midline, performance status, mental status, and localization of the tumor in an eloquent area [19-23]. Growth rate in time, even within the first six months is also of major prognostic significance [10]. Alternative imaging techniques also hold promise. PET imaging allows the identification of tumors in which a watch-and-wait policy may not be the right choice. Baseline amino acid uptake on (18)F-FET PET and a diffuse versus circumscribed tumor pattern on MRI were found to be strong predictors for the outcome of patients with low-grade glioma [24]. Moreover, maps of (18)FET uptake kinetics were found to correlate strongly with histopathology in suspected grade II gliomas [9]. How to implement these factors and imaging techniques optimally in the care of presumed LGG patients has not been investigated. Still, in the presence of multiple poor prognostic factors, it is unlikely that treatment in patients with nonenhancing LGG-like lesions can be postponed for a clinically relevant period. As a conclusion, a more active approach in patients with presumed or proven LGG over 45-50 years of age, with symptoms or signs other than seizures, or with larger and/or rapidly growing lesions is warranted. The value of PET scans needs to be further investigated in prospective cohorts.

Similar considerations apply for further adjuvant therapy after initial resection. Several studies have shown that residual disease after surgery is associated with a shorter time to radiological progression $[6,25]$. In subtotally resected low grade glioma patients under 40 years of age, the presence of residual disease ( $\geq 1 \mathrm{~cm}$ tumor) following surgery, initial tumor diameter over $4 \mathrm{~cm}$, and astrocytic histology proved to be poor prognostic factors for radiological progression [25]. Whether this implies that in the presence of postoperative residual tumor immediate adjuvant treatment is required is however a different matter: that will also depend on the rationale for surgery. For documented growth, mass effect, or deficits, an adjuvant treatment should be considered since these patients have an unfavorable prognostic profile. If however surgery was performed in a young patient with seizures only, the patient can be followed with further treatment when growth is radiologically documented.

In young patients, with a nonenhancing intracerebral lesion suspected for a low-grade glioma, without mass effect and without signs other than well-controlled seizures, a watch-and-wait policy can be followed provided the patient is carefully clinically monitored including MR follow-up. A reasonable policy is to make a first followup scan within 2-3 months of the first scan to detect the early progression of a high-grade tumor. In those cases that are being followed, histological confirmation can be postponed until the time the beginning of treatment is clinically indicated (e.g., in case of radiological progression, clinical deterioration, uncontrolled seizures).

\section{Treatments of low grade glioma}

\section{Surgery}

There are four objectives when performing surgery in suspected LGG: (1) histological confirmation of the nature of the lesion, (2) improvement of the neurological condition of the patient, (3) reducing the risk of tumor growth, and (4) prevention of malignant transformation. The first of these is an obvious one. Regarding the other objectives, retrospective series suggest that surgery may improve the neurological condition and the control of seizures $[18,26,27]$. There are no randomized trials in LGG on the impact of extent of resection on survival. Extensive data from uncontrolled studies suggest an improved outcome of LGG after early extensive resection. Without exception, all these studies are either retrospective surveys or more or less prospective cohort series in which patients were entered after surgery. The latter studies do not describe the outcome of similar patients that were managed conservatively. The impact of the bias that is inherent to the decision to operate (confounding by indication) is unknown, but one should realize that the large and excellent series from UCSF describing over 
200 operated patients mentions that more than 800 LGG patients were seen in that $p$ at the institution [6]. Moreover, all studies show that size and extension of LGG are independent prognostic factors, and an inverse correlation between extent of resection and size of the lesion has been documented [19]. As an example, it is unclear whether ill-defined and deep lesions, which are usually not considered ideal candidates for resection, have the same prognosis as more superficially located, clearly defined lesions. Because of their distinct growth pattern, a difference in molecular background is to be expected. A growing body of data demonstrates that prognosis in LGG depends on molecular profile up (in particular IDH1 mutations, MGMT promoter methylation, $1 \mathrm{p} / 19 \mathrm{q}$ co-deletion, TP53 mutations). Specifically, evidence is accumulating that the patients with IDH nonmutated tumors are older, and have tumors that are larger with a more infiltrative pattern on MRI. In contrast, tumors with IDH mutations may be more often localized in the frontal lobe and more often present with seizures [28, 29]. Such differences in molecular background will affect outcome, regardless of treatment, and the finding of a better prognosis after more extensive resection may-in part-be the consequence of these baseline differences in molecular profile. It underscores our limited knowledge to what extent early surgery has an impact on the natural behavior of LGG. Regardless of these considerations, all evidence supports a resection as extensive as safely possible once a surgery is planned. To obtain this goal, specialized procedures such as awake craniotomy, functional neuroimaging in patients with tumors in eloquent areas, and intraoperative MRI evaluation of extent of resection should be considered [30-32]. This allows a safer and more extensive resection, which may improve survival [32].

\section{Radiation therapy}

The efficacy of radiation therapy (RT) in low grade glioma has been demonstrated by a large randomized trial that showed an increase in time to progression after early RT in comparison to observation (and RT at the time of progression) [12]. Early radiotherapy (to a dose of 54 Gy in fractions of $1.8 \mathrm{~Gy}$ ) improved the median progression free survival from 3.4 to 5.3 years. As most patients in the observational arm received ('salvage') radiotherapy at the time of recurrence, no effect on overall survival was seen-further supporting the role of RT in this disease. The overall picture that emerges from this trial is that the timing of radiotherapy is less relevant as long as it is given. The trial did not investigate whether early RT helps to maintain the clinical condition of the patients, but at one year the seizures were better controlled in the RT arm. Another prospective trial observed a clear radiological response to RT in almost one third of patients, and small retrospective surveys have suggested improvement of neurological function or improved seizure control after radiation $[17,20]$. Because even after involved field irradiation, virtually all recurrences of LGG occur within the irradiated volume, one might expect a better local control after a higher dose of irradiation. However, two large randomized multicenter trials totaling 590 patients failed to detect improved survival after 59.4-64.8 Gy as compared to $45-50.4$ Gy [20,33]. Currently, it is advised to treat these tumors with involved field RT to a dose of $50.4-54$ Gy in fractions of $1.8 \mathrm{~Gy}$.

\section{Chemotherapy}

The role of chemotherapy in LGG is still incompletely understood. The results of the randomized phase III RTOG study on adjuvant PCV chemotherapy after RT are still pending. At the most recent presentation of the outcome, adjuvant PCV after RT was reported to increase PFS but not OS [34]. The data from the randomized EORTC study (radiotherapy versus chemotherapy in patients with LGG requiring treatment) will take some more years to mature. The currently available studies are uncontrolled phase II studies with more recent studies describing activity of temozolomide and older studies exploring PCV. Response assessment is challenging in these slow growing nonenhancing tumors: responding tumors may show only minimal decreases and only after the end of the treatment [35-37]. In small series, it has been suggested that PET imaging with radioactively labeled aminoacids may identify responding patients early on [38]. These studies report efficacy of both PCV and temozolomide, with more frequent responses and longer duration of response in 1p/19q co-deleted tumors [36, 39, 40]. With temozolomide, the reported median time to progression in the entire cohort was 28 months. In $1 p / 19 q$ co-deleted tumors response may, however, last many more years [36, 40]. Astrocytoma may also respond, usually with 'minor' responses but with often clinically interesting disease stabilization. More than half of the patients suffering from dedifferentiated astrocytoma relapsing after radiotherapy responded to temozolomide; 6 months PFS in this group was $67 \%$, and the median overall survival was 14 months [41]. Taken together, these data confirm the role of chemotherapy for these patients, leaving the question of timing still unanswered: Chemotherapy first? Or at recurrence after RT? Or in combination with RT? The tendency to use chemotherapy in lieu of RT in larger lesions especially when sensitivity to chemotherapy is expected (oligodendroglioma with combined $1 \mathrm{p} / 19 \mathrm{q}$ loss) is intuitively attractive to delay RT (and inherent late neurotoxicities), but good quality clinical data to guide decisions are lacking. Because of its better tolerability temozolomide has become the drug of choice, but current trials on glioblastoma have reminded physicians of the activity of nitrosourea's in glioma. These drugs including combination regimen (e.g., PCV) are therefore not to be forgotten. 


\section{Conclusion}

There are several treatment options for low grade glioma. The choice for early surgery in young and asymptomatic patients is in particular driven by the hope to improve survival. In particular, smaller, well circumscribed lesions in noneloquent regions appear good candidates for early aggressive surgery. If a conservative watch-andwait policy is followed, treatment should be considered in case of clear documented growth without waiting for the development of focal deficits. Uncontrolled seizures are a reason for treatment, as seizure control may improve with antitumor treatment. Especially in long term survivors, the use of radiotherapy is complicated by delayed effects on cognition, for which side effect must be balanced against effects of uncontrolled tumor growth on cognition. The best timing of chemotherapy versus radiotherapy is still unclear; the ongoing randomized trials must help clarify that. In case of larger lesions or chemotherapy responsive tumors, early chemotherapy should be considered.

\section{Conflict of interest}

JEB, TJS have no conflicts of interest. MJVDB has received honoraria from MSD.

\section{Open Access}

This article is distributed under the terms of the Creative Commons Attribution License which permits any use, distribution, and reproduction in any medium, provided the original author(s) and the source are credited.

\section{References}

1. Maintz D, Fiedler K, Koopmann J, et al. Molecular genetic evidence for subtypes of oligoastrocytomas. J Neuropathol Exp Neurol. 1997;56:1098-1104.

2. Douw L, Klein M, Fagel SS, et al. Cognitive and radiological effects of radiotherapy in patients with low-grade glioma: long-term follow-up. Lancet Neurol. 2009;8:810-18.

3. Reijneveld JC, Sitskoorn MM, Klein M, et al. Cognitive status and quality of life in suspected versus proven low-grade gliomas. Neurology. 2001;56:618-23.

4. Olson JD, Riedel E, DeAngelis LM. Long-term outcome of low-grade oligodendroglioma and mixed glioma. Neurology. 2000;54:1442-8.

5. Surma-aho O, Niemelä M, Vilkki J, et al. Adverse long-term effects of brain radiotherapy in adult low-grade glioma patients. Neurology. 2001;56:1285-90.

6. Smith JS, Chang EF, Lamborn KR, et al. Role of extent of resection in the long-term outcome of low-grade hemispheric gliomas. J Clin Oncol. 2008;26:1338-45.

7. Kondziolka D, Lunsford LD, Martinez AJ. Unreliability of contemporary neurodiagnostic imaging in evaluating suspected adult supratentorial (low-grade) astrocytoma. J Neurosurg. 1993;79:533-6.

8. Mandonnet E, Delattre JY, Tanguy ML, et al. Continuous growth of mean tumor diameter in a subset of grade II gliomas. Ann Neurol. 2003;53:524-8.
9. Kunz M, Thon N, Eigenbrod S, et al. Hot spots in dynamic (18)FET-PET delineate malignant tumor parts within suspected WHO grade II gliomas. Neuro Oncol. 2011;13:307-16.

10. Brasil CG, Ciccarelli O, Altmann DR, et al. Low-grade gliomas: six-month tumor growth predicts patient outcome better than admission tumor volume, relative cerebral blood volume, and apparent diffusion coefficient. Radiology. 2009;253:505-12.

11. Pallud J, Capelle L, Taillandier L, et al. Prognostic significance of imaging contrast enhancement for WHO grade II gliomas. Neuro Oncol. 2009;11:176-82.

12. Van Den Bent MJ, Afra D, De Witte O, et al. Long term results of EORTC study 22845: a randomized trial on the efficacy of early versus delayed radiation therapy of lowgrade astrocytoma and oligodendroglioma in the adult. Lancet. 2005;366:985-90.

13. Taphoorn MJB, Klein Schiphorst A, Snoek FJ, et al. Cognitive functions and quality of life in patients with lowgrade gliomas: the impact of radiotherapy. Ann Neurol. 1994;36:48-54.

14. Correa DD, Shi W, Thaler HT, et al. Longitudinal cognitive follow-up in low grade gliomas. J Neurooncol. 2008;86:321-7.

15. Klein M, Heimans JJ, Aaronson NK, et al. Effect of radiotherapy and other treatment-related factors on mid-term to long-term cognitive sequelae in low grade gliomas: a comparative study. Lancet. 2002;360:1361-8.

16. Rees J, Watt H, Jager HR, et al. Volumes and growth rates of untreated adult low-grade gliomas indicate risk of early malignant transformation. Eur J Radiol. 2008;21(1):54-64.

17. Soffietti R, Borgogne M, Ducati A, et al. Efficacy of radiation therapy on seizures in low-grade astrocytomas. NeuroOncology. 2005;7:389 (abstract \#422, abstr).

18. Chang EF, Potts MB, Keles GE, et al. Seizure characteristics and control following resection in 332 patients with lowgrade gliomas. J Neurosurg. 2008;108:227-35.

19. Pignatti F, Van Den Bent MJ, Curran D, et al. Prognostic factors for survival in adult patients with cerebral low-grade glioma. J Clin Oncol. 2002;20:2076-84.

20. Shaw E, Arusell RM, Scheithauer B, et al. A prospective randomized trial of low versus high dose radiation in adults with a supratentorial low grade glioma: initial report of a NCCTG-RTOG-ECOG study. J Clin Oncol. 2002;20:2267-76.

21. Brown PD, Buckner JC, O'Fallon JR, et al. Importance of baseline mini-mental state examination as a prognostic factor for patients with low-grade glioma. Int J Radiation Oncol Biol Phys. 2004;59:117-25.

22. Chang EF, Smith JS, Chang SM, et al. Preoperative prognostic classification system for hemispheric low-grade gliomas in adults. J Neurosurg. 2008;109:817-24.

23. Daniels TB, Brown PD, Felten SJ, et al. Validation of EORTC prognostic factors for adults with low-grade glioma: a report using intergroup 86-72-51. Int J Radiat Oncol Biol Phys. 2011;81:218-24.

24. Floeth FW, Pauleit D, Sabel M, et al. Prognostic Value of O-(2-18 F-Fluoroethyl)-L-Tyrosine PET and MRI in LowGrade Glioma. J Nucl Med. 2007;48:519-27.

25. Shaw EG, Berkey B, Coons SW, et al. Recurrence following neurosurgeon-determined gross-total resection of adult supratentorial low-grade glioma: results of a prospective clinical trial. J Neurosurg. 2008;109:835-41.

26. Gunnarsson T, Olafsson E, Sighvatsson V, et al. Surgical treatment of patients with low-grade astrocytomas and medically intractable seizures. Acta Neurol Scand. 2002;105:289-92. 
27. Duffau H, Capelle L, Lopes M, et al. Medically intractable epilepsy from insular low-grade gliomas: improvement after an extended lesionectomy. Acta Neurochir (Wien). 2002;144:563-72.

28. Metellus P, Coulibaly B, Colin C, et al. Absence of IDH mutation identifies a novel radiologic and molecular subtype of WHO grade II gliomas with dismal prognosis. Acta Neuropathol. 2010;120:719-29.

29. Stockhammer F, Misch M, Helms HJ, et al. IDH1/2 mutations in WHO grade II astrocytomas associated with localization and seizure as the initial symptom. Seizure. 2012;21(3):194-7.

30. Duffau H, Lopes M, Arthuis F, et al. Contribution of intraoperative electrical stimulations in surgery of low grade gliomas: a comparative study between two series without (1985-96) and with (1996-2003) functional mapping in the same institution. J Neurol Neurosurg Psychiatry. 2005;76:845-51.

31. Claus E, Horlacher A, Hsu L, et al. Survival rates in patients with low-grade glioma after intraoperative magentic resonance image guidance. Cancer. 2005;103:1227-33.

32. Chang EF, Clark A, Smith JS, et al. Functional mapping-guided resection of low-grade gliomas in eloquent areas of the brain: improvement of long-term survival. Clinical article. J Neurosurg. 2011;114:566-73.

33. Karim ABMF, Maat B, Hatlevoll R, et al. A randomized trial on dose-response in radiation therapy of low grade cerebral glioma: European Organization for Research and Treatment of Cancer (EORTC) study 2284. Int J Radiation Oncol Biol Phys. 1996;36:549-56.

34. Shaw EG, Wang M, Coons S, et al. Final report of Radiation Therapy Oncology Group (RTOG) protocol 9802: Radiation therapy (RT) versus RT + procarbazine, CCNU, and vincristine (PCV) chemotherapy for adult low-grade glioma (LGG). Proc Am Soc Clin Oncol. 2008;26 (abstract 2006, abstr).
35. Peyre M, Cartalat-Carel S, Meyronet D, et al. Prolonged response without prolonged chemotherapy: a lesson from PCV chemotherapy in low-grade gliomas. Neuro Oncol. 2010;12:1078-82.

36. Kaloshi G, Benouaich-Amiel A, Diakite F, et al. Temozolomide for low-grade gliomas: predictive impact of $1 \mathrm{p} / 19 \mathrm{q}$ loss on response and outcome. Neurology. 2007;68:1831-6.

37. Van Den Bent MJ, Wefel JS, Schiff D, et al. Response assessment in neuro-oncology (a report of the RANO group): assessment of outcome in trials of diffuse low-grade gliomas. Lancet Oncol. 2011;12:583-93.

38. Wyss M, Hofer S, Bruehlmeier M, et al. Early metabolic responses in temozolomide treated low-grade glioma patients. J Neurooncol. 2009;95:87-93.

39. Buckner JC, Gesme D, O'Fallon JR, et al. Phase II trial of procarbazine, lomustine, and vincristine as initial therapy for patients with low-grade oligodendroglioma or oligoastrocytoma: efficacy and and associations with chromosomal abnormalities. J Clin Oncol. 2003;21:251-5.

40. Biemond-ter Stege E, Kros JM, de Bruin HG, et al. Treatment of low grade oligodendroglial tumors with PCV chemotherapy. Cancer. 2005;103:802-9.

41. Taal W, Dubbink HJ, Zonnenberg CB, et al. First-line temozolomide chemotherapy in progressive low-grade astrocytomas after radiotherapy: molecular characteristics in relation to response. Neuro Oncol. 2011;13:235-41. 